Studies that link obesity to poor PF have typically been crosssectional, limited to two time-points or relied on retrospectively recalled height and weight. We aimed to establish associations between PF at age 50y and i) birthweight and BMI across the life-course; ii) BMI gains at specific life-stages and iii) age of obesity onset.

Methods The 1958 birth cohort includes all born in one week, in March 1958, across Britain. BMI (kg/m2) was calculated ( $\mathrm{N}=4,173$ (males); 4,501 (females)) using height and weight measured at school $(7,11,16 y)$, in participant's homes $(33,45 \mathrm{y})$ or self-reported $(23,50 \mathrm{y})$. PF at $50 \mathrm{y}$ was assessed via postal questionnaire using the validated $\mathrm{PF}$ subscale of the Short-form 36 (SF-36) survey. The lowest sex-specific 10th centile were defined as poor PF. Missing data was imputed via multiple imputation. Associations were examined using logistic regression, adjusting for social class, education and health behaviours.

Results Birthweight was not associated with PF. At each adult age, odds of poor PF were highest for obese (vs normal) e.g. for $23 \mathrm{y}$ obesity the $\mathrm{OR}_{\text {adjusted }}$ for poor $\mathrm{PF}$ was 2.28 $(1.34,3.91)$ and $2.67(1.72,4.14)$ in males and females respectively. BMI gains from adolescence were related to poor PF, e.g. for females, $\mathrm{OR}_{\text {adjusted }}$ per $\mathrm{SD}$ in $\mathrm{BMI}$ gain between 16$23 y$ was $1.28(1.13,1.46)$; for BMI gains $45-50 y$ it was 1.36 $(1.11,1.65)$. Mean BMI at 50y increased with earlier onset of obesity; e.g. in males, from $31.4 \mathrm{~kg} / \mathrm{m}^{2}$ for mid-adult onset to $35.1 \mathrm{~kg} / \mathrm{m}^{2}$ for child onset. Longer duration of obesity was associated with poor PF (p-trend<0.001), e.g. in males, for childhood obesity onset (vs. never obese) $\mathrm{OR}_{\text {adjusted }}$ was 2.32 $(1.26,4.29)$; for mid-adulthood obesity onset it was $1.50(1.16$, 1.96); associations were abolished with further adjustment for $50 \mathrm{y}$ BMI.

Conclusion Study strengths include the large nationwide cohort followed from birth and prospective measures of BMI and PF, albeit BMI at some ages was self-reported. Obesity, BMI gains and earlier obesity onset were associated with poor $\mathrm{PF}$ in mid-adulthood. Findings relating to duration of obesity are important given the increasing prevalence of childhood obesity, which tends to track into adulthood. Our study highlights the importance of preventing and delaying obesity onset to mitigate the risk of poor PF in mid-adulthood.

\section{P50 PREDICTIVE FACTORS FOR INDETERMINATE RESULTS IN INTERFERON- $\gamma$ RELEASE ASSAYS - A PROBLEM TO CONSIDER}

\begin{abstract}
1,2,3 JA Santos* ${ }^{*}, 5 \mathrm{R}$ Duarte, ${ }^{1,3} \mathrm{C}$ Nunes. ${ }^{1}$ Escola Nacional de Saúde Pública, Universidade NOVA de Lisboa; ${ }^{2}$ Instituto Nacional de Saude Dr. Ricardo Jorge, Lisboa, Portugal; ${ }^{3}$ Centro de Investigação em Saúde Pública, Lisboa, Portugal; ${ }^{4}$ Faculdade de Medicina da Universidade do Porto, Porto, Portugal; ${ }^{5}$ Centro Diagnóstico Pneumológico de Vila Nova de Gaia, Porto, Portugal
\end{abstract}

\subsection{6/jech-2019-SSMabstracts.201}

Background Interferon- $\boldsymbol{\gamma}$ release assays (IGRA) are a crucial diagnostic tool for the detection of a Mycobacterium tuberculosis infection in order to control and eliminate the tuberculosis (TB) epidemic. Indeterminate results can occur and represent a considerable problem for clinical management, since they imply the lack of clear information about the patient's TB infection status. The aim of the study was to identify risk factors that could be associated with indeterminate IGRA results.
Methods Retrospective cohort study carried out using data from the Portuguese National Tuberculosis Surveillance system, from 2008 to 2015 . Were included in the study 1230 patients with active TB and an IGRA result. The IGRA test used in the patients enrolled in the study was the QuantiFERON-TB Gold In-Tube (Qiagen). The association between indeterminate IGRA results and sociodemographic factors, comorbidities and the site of disease were evaluated through bivariate and multivariate logistic regression analysis.

Results Of the 1230 patients reported with active TB in the SVIG-TB database (2008-2015) that underwent an IGRA test, 857 patients $(69.7 \%)$ had a positive test result, $212(17.2 \%)$ had a negative result and 161 (13.1\%) had an indeterminate result. Majority of the patients with indeterminate results were male $(67.7 \%)$ and more than half had more than 50 years (57.1\%). The proportion of indeterminate results increased as the age increased, with patients over 80 years old presenting the highest proportion of indeterminate results. Age $\geq 65$ years (OR 2.51, p<0.001), alcohol abuse (OR 3.04, p=0.001) and pulmonary TB (OR 3.07, p<0.001) were predictive factors for indeterminate IGRA results.

Conclusion Age $\geq 65$ years, alcohol abuse and pulmonary TB were identified as factors for the occurrence of indeterminate IGRA results. The first two factors can be identified prior to the test and thus help to quickly identify the probable cause of an indeterminate outcome and lead to the use of other clinical and diagnostic means to detect a possible infection.

\section{P51 SENSITIVITY AND AGREEMENT OF AN INTERFERON- GAMMA RELEASE ASSAY AND TUBERCULIN SKIN TEST IN PATIENTS WITH PULMONARY AND EXTRAPULMONARY TUBERCULOSIS}

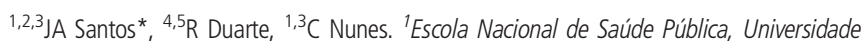
NOVA de Lisboa, Portugal; ${ }^{2}$ Instituto Nacional de Saude Dr. Ricardo Jorge, Lisboa, Portugal; ${ }^{3}$ Centro de Investigação em Saude Publica, Lisboa, Portugal; ${ }^{4}$ Faculdade de Medicina da Universidade do Porto, Porto, Portugal; ${ }^{5}$ Centro Diagnóstico Pneumológico de Vila Nova de Gaia, Porto, Portugal
\end{abstract}

\subsection{6/jech-2019-SSMabstracts.202}

Background Tuberculosis (TB) is still a serious global public health concern, being essential a rapid and accurate diagnosis of infected individuals. The aim of this study was to estimate the sensitivity of the interferon- $\gamma$ release assays (IGRA) and tuberculin skin test (TST) in patients diagnosed with active TB and the agreement between the tests.

Methods Retrospective cohort study carried out using data from the Portuguese National Tuberculosis Surveillance system, from 2008 to 2015. The study included all TB cases with an IGRA and TST result $(n=727)$. The IGRA test used in the patients enrolled in the study was the QuantiFERON-TB Gold In-Tube (Qiagen). Sensitivity was calculated with 95\% confidence interval $(95 \% \mathrm{CI})$ for each test separately and in combination (IGRA and TST-5 mm or IGRA and TST-10 mm) and outcomes were compared using McNemar's test. Kappa coefficient $(\mathrm{k})$ was used to evaluate the agreement between IGRA and TST test results.

Results The mean age of the patients was 47.9 years ( \pm standard deviation 20.0 years), ranging from less than a 1 year to 91 years, with the age group 16-64 years representing the majority of cases. IGRA, TST-5 mm and TST-10 mm were positive in $82.4 \%, 84.5 \%$ and $78.4 \%$ of the $\mathrm{TB}$ patients. These results imply that $128(17.6 \%), 112(15.4 \%)$ and 157 